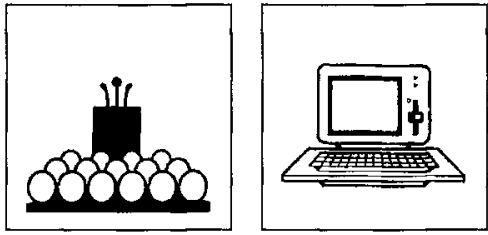

Was ist SIJUS-STRAF-STA?

Entwicklungsgeschichte

50 Installationen

Hard- und Softwareausstattung

Entwicklungsschwerpunkt bisher

Funktionsumfang

Oberstaatsanwalt Heinz Hoynatzky, Landshut, ist Leiter der Abteilung Geschätsstellenautomation in Straft sacben bei Staatsanwaltschaften und Gerichten in Bayern.

\title{
SIJUS-Straf-Staatsanwaltschaft
}

\section{Heinz Hoynatzky}

SIJUS-STRAF-STA ist ein Geschäftsstellenprogramm, das stufenweise realisiert, in seiner Endstufe alle Arbeitsbereiche der Staatsanwaltschaft, d.h. von der Übernahme der Daten von der Polizei bis zur Aktenausscheidung unterstützt.

Mit der Entwicklung wurde 1987 in einer gemeinsamen Arbeitsgruppe der Länder Niedersachsen und Bayern mit damals SIEMENS, heute SIEMENS-NIXDORF, begonnen. Heute sind an der Arbeitsgruppe noch die Länder Baden-Württemberg und Bremen beteiligt, über den Anwenderkreis darüber hinaus die Länder Sachsen, Sachsen-Anhalt und das Saarland. Ein weiteres Land wird in den nächsten Tagen hinzukommen.

Bis Ende des Jahres wird das Programm in 50 Staatsanwaltschaften eingesetzt sein, z.Zt. sind es 45. Die Programmvorgaben entstehen in einer Arbeitsgruppe, der Vertreter des mittleren, gehobenen und höheren Dienstes aus Behörden verschiedener Größenordnungen angehören.

\section{Technische Daten}

Hardware:

In den beteiligten Ländern wird das Programm auf Maschinen der Reihe

- MX300 und MX500, jeweils INTEL, sowie

- RM200 RM400 und RM600, jeweils RISC,

mit SINIX-kompatiblen Bildschirmeinheiten betrieben.

Als Drucker werden LED-Drucker 9022, 4819, 4820, 4821 mit Barcodedruck und in der Regel 2 Schächten, 1 Schacht für Etiketten oder Sonderdrucke, z.B. Postzustellungsurkunden, eingesetzt.

\section{Software:}

Für die Anwendung wird nur Standardsoftware eingesetzt, z.Zt.

- SINIX V5.41

- INFORMIX-SE/ONLINE (SINIX) V4.1

- INFORMIX-NET/STAR (SINIX) V4.1 im Netzbetrieb

- HIT V4.1B mit gerade laufender Umstellung auf HIT V5.1

Für den Anwender sind keine DV-technischen Kenntnisse erforderlich. Der Systemverwalter benötigt Kenntnisse im Rahmen der SINIX-Systembetreuung.

Der Entwicklungsschwerpunkt lag bisher ausschließlich in den Bereichen

- Geschäftsstelle (Verfolgung und Vollstreckung)

- Schreibkanzlei

und erstreckt sich parallel nunmehr auch auf den höheren Dienst einschließlich der Wirtschaftsfachkräfte, wobei hier die Einbindung von Arbeitsplatzcomputern (PC) erprobt wird. Näheres darüber wird im weiteren Verlauf Herr Janovsky berichten, der diese Entwicklung in Bayern verantwortlich betreibt und in Hof pilotiert.

Das Programm SIJUS-STRAF-STA deckt unter dem Gesichtspunkt der Einmalerfassung von Daten alle Arbeitsfunktionen einer Staatsanwaltschaft ab, d.h.

- Erfassung von Verfahren aller Registerzeichen,

- Aktenanlage mit Etiketten und Vorverfahrenslisten, Mitteilungsschreiben an Geschädigten,

- Akten- und Wiedervorlagen-/Fristenkontrolle,

- StA-Statistik (Zählkarte, Monatsübersicht),

- Anfragen und Mitteilungen an das BZR/VZR/GZR/AZR,

- Personenfahndung (BZR, INPOL, SIS, SIS und europäische Nachbarstaaten, INTERPOL),

- Strafverfolgungsstatistik,

- MiStra und sonstige Mitteilungspflichten,

- Geldstrafen- und Kostenvollstreckung, in Sachsen und Bayern mit Leitung zur Landesjustizkasse und damit jeweils aktueller Kontoübersicht,

- Datenlöschung manuell und automatisch, 
- Datensperre von Personen und Verfahren aus ermittlungstaktischen oder datenschutzrechtlichen Gründen,

- Phonetische, Stern- und Suche mit verknüpften Daten nach Personen und Verfahren,

- Sammeleingabefunktionen für identische Arbeitsschritte mit Barcodelesern,

- Wiedervorlageübersichten täglich oder nach einstellbaren Daten oder Zeiträumen,

- Monierungsschreiben für versandte Akten automatisch oder manuell,

- Unterstützung der Teil- und Aussonderung von Akten oder Abgabe an das Staatsarchiv,

- Textverarbeitung als kleines und großes Schreibwerk mit Zugriff auf vorhandene Daten,

- Kürzeleingabe für häufig vorkommende Aktenempfänger oder Verfahrensbeteiligte, z.B. Rechtsanwälte, Behörden, Versicherungen u.a.,

- Schnittstelle für den Empfang externer Daten (z.B. Polizei) und den Versand von Daten,

- Datenbankabfragen und -auswertungen mit standardmäßigen INFORMIX-Programmen wie

$\begin{array}{ll}\text { PERFORM } & \text { (Formatprogramm) } \\ \text { ACEGO } & \text { (Listenausdruck) } \\ \text { RDSQL } & \text { (Dialogprogramm) } \\ \text { FORMBUILD } & \text { (Formaterstellung) } \\ \text { ACEPREP } & \text { (Listenformaterstellung) }\end{array}$

- Jederzeit möglicher Aufruf des aktuellen Status des Referats/Dezernats (Eingabe, Erledigungen, Sitzungsstunden, eigene Ermittlungstätigkeit usw.)

Die Datenhaltung findet auf einem zentralen Rechner statt, mit dem die Anwender je nach Größe der Behörde direkt, über TACSI, TACLAN, einen oder mehrere Rechner innerhalb eines Hauses und über Postleitungen (ISDN) mit Außen- und Zweigstellen vernetzt sind.

Durch das Programm werden den Geschäftsstellen Routinearbeiten abgenommen oder wesentlich erleichtert. Jede anfallende Arbeit kann von jedem Arbeitsplatz aus erledigt werden. Bisher notwendige Aufteilungen der Arbeiten in verschiedene Organisationseinheiten werden weitgehend überflüssig, so daß der Aktenumlauf reduziert werden kann. Die Verfahrensbearbeitung wird beschleunigt.

Es gibt keine isolierten Dateneingaben, d.h. Daten, die wichtige Informationen enthalten, stehen sofort für alle zur Verfügung. Dadurch sind die Daten immer aktuell, zur Zeit allerdings nur für die einzelne Staatsanwaltschaft, in Sachsen und Bayern ab Oktober durch Aufbau eines Datennetzes aber auch für andere Staatsanwaltschaften im Land. Die zentralen Register werden mit Einleitung der Vollstreckung durch ineinandergreifende Funktionen sofort unterrichtet.

Die Schulung der Anwender wird in Wochenkursen vor Ort vorgenommen, die der Systemverwalter und der Anwender, die tiefer einsteigen wollen, in der EDV-Schule in Wildeshausen (Niedersachsen), Radebeul (Sachsen) und Pegnitz (Bayern).

Die Programmentwicklung ist nicht abgeschlossen. Die Funktionalität wird laufend erweitert, die vorhandene Funktionalität ständig optimiert und der Hard- und Softwareentwicklung angepaßt. Dies ist möglich und trotz der Mächtigkeit des Programms auch finanzierbar, weil 7, bald 8 Partner sich die Kosten teilen und damit für jedes Land nur Kostenanteile entstehen, die bei vergleichbarer Funktionalität deutlich unter denen einer einzelnen Landeslösung liegen.

Zentrale Datenvorbaltung

Arbeitserleichterung

Zentralisierte Datenpflege

Mitarbeiterschulung

Programmentwicklung 